\title{
SPECKLE V NON-REDUNDANT MASKING
}

\author{
C.A. HANIFF* \\ MRAO, Cavendish Laboratory, \\ Madingley Road, Cambridge, \\ CBS OHA, UK
}

\begin{abstract}
Unlike their radio counterparts, optical astronomers have paid relatively little attention to the role of the aperture function when designing their high resolution imaging experiments. This is despite the fact that it can be easily modified with the use of a suitable pupil plane mask. While the defining characteristics of the two most favoured pupil configurations have never been in doubt - negligible atmospheric noise in the case of a non-redundant configuration of small holes and high photon rates for a filled pupil - the relative merits of these two choices in terms of imaging performance in the presence of turbulence appear not to have been carefully investigated until very recently.

Most existing comparisons of fully-filled aperture (FFA) and non-redundant mask (NRM) based imaging strategies appear to have ignored a number of fundamental and practical difficulties that are often encountered in practice. In the intermediate regime, between very high and very low light levels, that characterizes most astrophysical applications hybrid imaging schemes seem most profitable. These utilize partially-redundant pupil geometries that combine the advantages of redundancy at low light levels without incurring the penalties associated with fully redundant beam recombination. Such pupil geometries are also useful in reducing the level of systematic effects that often plague speckle imaging experiments.
\end{abstract}

Key words: Speckle - non-redundant-masking - aperture distributions - partially redundant pupils

\section{Introduction}

Since the introduction of bispectral imaging techniques in the mid 1980's the relative merits of FFA and NRM imaging methods have been the subject of much scrutiny [1]. As judged by the speckle imaging experiments reported in the literature the upshot of these studies has been to identify FFA techniques as being superior, and only a handful of workers have continued to utilize sparse pupil geometries regularly. However the majority of these comparisons have concentrated on issues that bear little relation to successful image recovery and invariably ignore the practical constraints that often represent the most significant limitations to astrophysical interferometric imaging experiments.

In this contribution we shall take a more pragmatic approach and recast the question 'Speckle $\mathbf{v}$ NRM' in a form that asks 'Which of these methods can recover the better image under realistic observing conditions?' Our conclusions may challenge conventional wisdom but hopefully will provide useful guidelines for the optimization of future interferometric imaging experiments.

\section{FFA v NRM - Current Opinions}

At first sight the differences between the FFA and NRM methods appear so obvious that it seems difficult to understand how there could ever have been any question as to their relative merits. However, although the features summarized in the table below have never been in question their effects on imaging performance have been

* also at Institute of Astronomy, Madingley Road, Cambridge, CB3 OHE, UK 
assessed in a variety of inappropriate ways. For example, most of the apparently undesirable features of the NRM method (such as its limited use of the pupil area) can have very little effect in terms of imaging performance under realistic observing conditions. It is this type of misunderstanding as to what the important design

\begin{tabular}{lll}
\hline & FFA & NRM \\
\hline Detected flux & Collects all & Collects small fraction \\
UV coverage & Complete & Sparse \\
Pupil type & Highly redundant & Non-redundant \\
\hline
\end{tabular}

criteria for interferometric experiments are that has polarized workers in the field and led to the necessity of a contribution such as this one. In order to redress this state of affairs we need to examine the repercussions of the pupil geometry, not in terms of quantities that may be relevant in conventional imaging, for example the detected flux, but in terms more appropriate to interferometry, and in particular with due regard to the practical limitations experienced at observatory sites. Hopefully the following sub-sections will clarify the debate somewhat.

\subsection{UV Plane Coverage \& Imaging Fidelity}

Given the historical dominance of filled-pupil telescopes at optical wavelengths, optical astronomers have been wary of accepting the sparse UV coverage that typifies the NRM method. Three main issues have caused the most confusion.

The first of these is simply the Fourier plane coverage required to map a source of given complexity. Experience at radio wavelengths has shown that the number of UV data required is approximately equal to the number of filled resolution elements in the restored image. Apart from the sun, for all astrophysical sources that have been suitable targets for interferometric imaging this number has been surprisingly small $(\leq 100)$, which implies that very dense UV coverage is not a necessary condition for the success of a typical interferometric imaging experiment. Despite the large body of empirical evidence supporting this claim, the completeness of the UV coverage afforded by a filled pupil is still sometimes cited as an overwhelming advantage of this particular pupil geometry. Because NRM experiments are optimized when the number of sub-apertures is small $(<10)[2]$ they are undoubtedly compromised because of their poor instantaneous Fourier plane coverage. However, the number of different array geometries required to sample the UV plane adequately is never very large and in most instances the dense UV coverage provided by a filled pupil is superfluous.

A more subtle point related to the UV coverage concerns the uniqueness and robustness of the imaging procedure itself. Because the inversion of NRM data uses the radio astronomical methods of self-calibration [3], the convergence properties of which are in principle model dependent, there have been questions raised about the reliability of NRM image reconstructions. This area has been studied in detail by radio astronomers in the context of comparisons between standard self-calibration 
and redundant-spacing calibration (RSC) [4]. Here RSC can be advantageous because it allows a single-step solution to the closure equations without any a priori knowledge of the sky brightness distribution. While this is certainly important for radio astronomers who are seeking extremely high dynamic range images $(\gg 100: 1)$, these questions of uniqueness have never been seen to be a problem in optical interferometric imaging where, in practice, image restoration is usually limited by the reliability of the Fourier data themselves.

Originally, continuity of the UV coverage was an important design goal for interferometric imaging experiments because of the use of recursive methods for Fourier phase recovery from the bispectrum [5]. Nowadays, the widespread adoption of global least-squares methods has made this requirement unnecessary, and so both sparse (NRM) and filled (FFA) pupil geometries can be exploited. Of course NRM bispectral data will still require additional a priori information for inversion because of the fundamental uncertainties in backprojecting the bispectral phases to the baseline phase space [6], but this is unlikely to be a significant drawback until attempts are made to image very complex sources.

In astronomical experiments the issues mentioned above can often be completely overridden by more practical considerations. Most importantly, for sources that vary rapidly (e.g. the sun or asteroids) the time taken to sample the UV plane adequately with a non-redundant pupil may exceed the variability timescale of the source and so the use of a filled pupil may be the only way to proceed. This type of constraint is far too often ignored in more theoretical discussions and is an indication of the pragmatic issues that need to be addressed in any realistic comparison of the FFA and NRM methodologies.

\subsection{Limiting Sensitivity}

Fundamental limiting sensitivity calculations have often been used to provide a useful discriminant between the FFA and NRM methods. As measured in these terms NRM experiments unquestionably fall far behind their FFA counterparts. It is straightforward to understand this in heuristic terms by considering measurements of the bispectrum at low light levels. Although the imaging of complicated objects requires knowledge of both the power spectrum and bispectrum, the overriding importance of Fourier phase recovery suggests that, at least in terms of fundamental limitations, we should restrict attention to the bispectrum. Note that in this regime the behaviour of other lower order spectra that may be useful for image recovery (e.g. the Knox-Thompson spectrum) is similar to that of the bispectrum, and so any conclusions we draw will be quite general.

If we first consider an NRM experiment using a mask comprising three $r_{o}$-sized holes it is straightforward to show that for an instantaneous bispectrum measurement to have any meaning at least 3 photons must be detected. Since the low light level $\mathrm{S} / \mathrm{N}$ of these bispectrum measurements will scale as $\left(n / N_{a}\right)^{3 / 2}$, where $n$ is the number of photons detected per sub-aperture per interferogram, and $N_{a}$ is the number of sub-apertures, the limiting sensitivity of NRM imaging will basically be reached when a single photon is detected per sub-aperture per integration time. The limiting sensitivity of FFA imaging (for image plane interferometers) will be 
governed by a similar constraint, in that at least three photons will have to be detected per interferogram. However, because of the much larger collecting area of the pupil, this sensitivity limit will correspond to a much fainter source.

For typical interferometric observations at a $5 \mathrm{~m}$ class telescope this sort of argument would imply a sensitivity advantage of $\sim 500$ for FFA methods. However, the results presented in the literature to date clearly demonstrate that such improvements are never realized. There appear to have been no successful image reconstructions that even begin to approach the diffraction-limit where the detected photon rate has been below $\sim 0.05$ per coherence area per integration time. This is completely understandable: at lower light levels most of the bispectral measurements will have such a low instantaneous $\mathrm{S} / \mathrm{N}$ that the number of interferograms required to achieve a useful $\mathrm{S} / \mathrm{N}$ will exceed the number that can be recorded in a night. An examination of the successful FFA image restorations also reveals that the dynamic ranges achieved in these reconstructions are usually quite low $(\sim 20: 1)$ and certainly worse than those attained in low-light level NRM imaging experiments.

Our conclusion then is that it is unreasonable to judge FFA methods as superior based solely on arguments that deal with fundamental sensitivity: the practical limitations imposed by a finite observing time allocation, and the empirical evidence of the difficulties associated with image recovery from low $\mathrm{S} / \mathrm{N}$ bispectral data are both constraints that must be assessed in parallel with these more fundamental issues.

\subsection{High-Light-Level Behaviour}

At sufficiently high light levels it is well established that the $\mathrm{S} / \mathrm{N}$ of filled aperture image plane interferometry saturates because of atmospheric noise [7]. NRM methods suffer no such limitation and under these conditions can exploit large numbers of sub- $r_{o}$ sized sub-apertures. Such NRM pupil configurations provide good instantaneous UV coverage and allow, in principle, the recovery of diffraction-limited images from single interferograms. Data obtained in this manner are also less affected by fluctuations in the mean seeing between observations of source and calibrator than are FFA data. There is thus quite general agreement that at least in this regime, which is albeit relatively rare for astrophysical observations, NRM methods are superior to image plane FFA techniques.

This $\mathrm{S} / \mathrm{N}$ saturation is a consequence of the redundant beam combination that characterizes FFA image plane interferometry and which leads to the mixing of incoherent contributions towards the same Fourier component. The use of a nonredundant mask obviously eliminates this problem, but at the expense of the effective collecting area of the pupil. A more satisfactory solution, that utilizes all of the pupil area while maintaining a non-redundant beam recombination, is to use a pupil plane interferometer. The merits of such a scheme have recently been reviewed in detail [8] but basically in the high-light-level regime they permit the use of the whole pupil without random attenuation of the transfer function. It is surprising that such an advantageous technique has been so little exploited for astrophysical imaging of bright sources. 


\subsection{LOW-LIGHT-LEVEL BehaViour}

Similar arguments to those used to justify FFA methods in terms of limiting sensitivity can be used to support claims that its low-light level performance is superior to NRM imaging. At worst these simply proffer increased flux collection and better UV coverage as undeniable merits. While these features cannot be denied, their effects on practical image recovery demand careful scrutiny. Because of the nonlinear methods utilized to reconstruct images from bispectral data it is non-trivial to derive the signal-to-noise ratios of these images from estimates of the $S / N$ of individual bispectrum measurements. An alternative method of comparison, and the one that we shall take here, is to examine the $\mathrm{S} / \mathrm{N}$ of the bispectrum measurements themselves, though with regard to both their number and nature, and incorporating any other practical details that may override theoretical considerations.

One possibility is to make a pointwise comparison of $S / N$. This is potentially inadequate because the nature of the bispectral measurements made with FFA and NRM techniques are quite different. NRM experiments sample the bispectrum very sparsely in a random fashion that explores the full extent of the bispectral hypervolume. The transfer function for this process is reasonably high, and so the $\mathrm{S} / \mathrm{N}$ of the data can be good even at low photon rates. In contrast FFA experiments permit the measurement of all of the bispectrum though with a highly attenuated transfer function. Only those regions of the bispectrum that suffer the least attenuation, and hence have the greatest $\mathrm{S} / \mathrm{N}$, are ever used for phase recovery, but these are precisely those regions (the so called near-axis locations) that are not sampled in NRM experiments.

Buscher [9] has made some progress towards addressing this issue, by comparing the reliability of Fourier phase determination using the near-axis bispectrum with the estimation of closure phases using NRM. This can be justified because the closure phases represent the primary phase observables in NRM image reconstruction whereas in FFA image reconstruction it is the recovered Fourier phases that take this role. His study finds the two techniques comparable despite the fact that the NRM configuration he examines collects only a small fraction of the incident flux from the source. On the basis of a single example he sensibly warns of drawing far reaching conclusions, but his results do suggest that a proper comparison of the two techniques requires more care than is usually taken.

A far more insidious type of argument in favour of FFA-based techniques involves the use of a global measure of the information content of the measured bispectrum. In particular, it is usually assumed that the total information in the bispectrum can be quantified by taking the product of a 'median' $S / N$ and the square root of the number of independent bispectral measurements. Using this measure it is straightforward to deduce that FFA methods must be preferred over NRM techniques by virtue of the huge number of independent bispectral points measured. While this procedure is defensible under certain restrictive conditions, for example if one is interested in the reliability of a measurement of the flux of an unresolved source [10], it bears little relation to the real problem at hand, that of diffraction limited image recovery.

This apparent discrepancy arises because most of the bispectral data collected in 
FFA imaging experiments are measured with extremely low $\mathrm{S} / \mathrm{N}$ even after integration over many hundreds of thousands of interferograms. There is now a large body of evidence, comprising both experimental results and those arising from numerical simulations [11], that suggests that it is in practice impossible to utilize bispectral data that have a $\mathrm{S} / \mathrm{N} \ll 1$ for image recovery. Although this empirical result has yet to be explained theoretically, it seems more than likely that it is a consequence of the modulo $2 \pi$ nature of the bispectrum phase and the non-linear algorithms used for image recovery: if this is so then it seems unreasonable to expect this limitation to be overcome in the near future.

Once the existence of such a hard bispectral $\mathrm{S} / \mathrm{N}$ threshold is accepted, the case for employing a highly redundant, but large area pupil becomes less persuasive. Although the number of power spectrum and bispectrum points accessible using FFA methods can be huge, this dense sampling is almost always achieved at the expense of the $\mathrm{S} / \mathrm{N}$ of the individual measurements. If data of $S / N \ll 1$ are not useful, it cannot be reasonable to justify FFA methods on these grounds alone. Unfortunately there have been few comparisons of FFA and NRM imaging undertaken where the same source has been 'observed' using both methods. Hopefully this discussion will stimulate, or perhaps provoke, someone to perform such an investigation so that once and for all a consensus can be reached on this matter.

\subsection{Systematic Effects}

Perhaps the most important area ignored in comparisons of FFA and NRM concerns the influence of systematic effects, most of which are well known by experienced astronomers. My aim here will not be to provide an exhaustive list, but rather to mention a few that can be particularly damaging to interferometric imaging experiments.

The most common of these is visibility (mis)calibration. Random fluctuations in the mean atmospheric conditions are often the limiting factor in determining the reliability of visibility amplitude measurements. FFA image plane configurations suffer most from this effect. The effects of seeing fluctuations can be ameliorated considerably by employing a non-redundant mask comprising sub- $r_{0}$ sized apertures, but this is only practical at high light levels. A more judicious solution is to utilize a FFA pupil plane interferometer. Such an instrument has a transfer function that is independent of the atmospheric phase aberrations [8] and so can be particularly advantageous if the seeing conditions are unstable. Telescope aberrations represent another hazard often experienced in actual imaging experiments. Once again, the FFA image plane configuration fares worse in this competition, with NRM and pupil-plane FFA based schemes improving on it in that order.

Arguably the most important obstacle to reliable interferometric imaging has been the lack of suitable noise-free detectors. CCD cameras have good sensitivity and linearity, but are difficult to operate successfully, i.e. with good readout noise performance, at low light levels with adequate time resolution. To some extent this has forced the use of photon counting cameras, which have much poorer quantum efficiencies and which can suffer from saturation effects at surprisingly low count rates. 
In many instances an appropriate pupil geometry has the potential to limit the effects of non-ideal equipment and unstable experimental conditions considerably, yet this possibility is very rarely exploited. Indeed, in certain instances, alternative courses of action have been pursued that have been instrumental in compromising, rather than enhancing, the measured Fourier data!

\section{Optimum Imaging Strategies - Partially Redundant Masking (PRM)}

As the above discussion has shown the cases for both FFA and NRM imaging can be cogently argued only under very restrictive conditions. At extremely high light levels NRM, or FFA/pupil plane methods are optimal. On the other hand, at the very lowest light levels FFA/image plane interferometry will always have a sensitivity advantage, although whether or not satisfactory image recovery will ever be possible in this regime is unclear.

Of much more interest is the intermediate regime in which almost all potential astrophysical sources lie, and in this case the discussion thus far has only served to highlight the inadequacies of both the FFA and NRM methods. However it should be clear that an optimal scheme would capitalize on the assets of both the FFA and NRM techniques, especially those that provide some measure of resistance to the systematic effects that can plague interferometric imaging experiments. A basic 'wish-list' might include good UV plane coverage, a reasonable collecting area, low redundancy in order to minimize atmospheric attenuation of the Fourier data, and as simple an experimental arrangement as possible. One possible approach is that of extended non-redundant-masking (ENRM) [2] in which the full pupil area is divided up into a number of non-redundant configurations each of which is analyzed separately. This satisfies some of the requirements mentioned above, but is certainly much more demanding in terms of instrumental complexity.

A more satisfactory solution is to modify the pupil geometry so as to 'tune' the response of the interferometric experiment to the particular astrophysical application. This type of apodization has recently been examined [12,13] for the cases of readout noise limited and photon counting detectors. These analyses investigated the effect of various apodizing masks on the $\mathrm{S} / \mathrm{N}$ ratios of power spectrum and bispectrum measurements, as well as their consequences in terms of UV coverage and resistance to seeing miscalibration. As an example of this type of analysis, we can consider two simple cases: a thin annular pupil and a long thin slit-type pupil. Both of these aperture geometries limit the flux detected from the source, which is often beneficial in terms of reducing the level of detector induced artefacts, but they differ markedly in other attributes that can affect their suitability for imaging. For example, the annular pupil provides complete instantaneous UV coverage, and is minimally redundant, whereas the linear pupil only samples the UV plane in one dimension and is highly redundant.

The $\mathrm{S} / \mathrm{N}$ ratios of power spectrum and bispectrum measurements made with these two pupils are also quite different: this is best exemplified using the expression for the instantaneous bispectral $\mathrm{S} / \mathrm{N}$ at low light levels. This scales as $(n / A)^{3 / 2} R_{t}$, where $A$ is the total pupil area, $n$ is the number of photons detected per coherence area per interferogram, and $R_{t}$ is the triplet redundancy of the bispectrum 
point being considered, i.e. the number of times the corresponding closure triangle is physically realized within the pupil. Two features of this expression warrant particular attention: first, that the total pupil area, $A$, appears in the denominator, so that for fixed $R_{t}$ increasing the pupil area will decrease the $\mathrm{S} / \mathrm{N}$, and second, that for any given source $n$ will remain constant regardless of the pupil configuration. It is easy to see that at low light levels a thin annular pupil will be heavily penalized because its area can be large yet its triplet redundancy is very low. On the other hand, a linear pupil of equivalent area may measure many bispectral points with high triplet redundancies, and so will be preferred. This scaling of the bispectral $\mathrm{S} / \mathrm{N}$ also highlights some of the shortcomings of the FFA and NRM methods: filled aperture experiments would be improved if the pupil area could be reduced without affecting $R_{t}$, whereas NRM experiments fail at low light levels precisely because $R_{t}=1$.

Within this framework a 1-dimensional partially redundant pupil - a long thin slit - appears to be an excellent compromise in terms of balancing UV coverage, $\mathrm{S} / \mathrm{N}$ and limiting sensitivity if experimental conditions, such as detector non-linearities, dictate a reduction in the detected photon rate. Indeed in certain instances this can provide power spectrum and bispectrum measurements with better $\mathrm{S} / \mathrm{N}$ than a filled pupil at all spatial frequencies higher than the seeing cutoff [13]. Interestingly the use of a pupil optimized in this way for interferometric imaging is not a new idea. Aime and his colleagues independently arrived at a similar conclusion in the late 1970's based on considerations of the $\mathrm{S} / \mathrm{N}$ of power spectral measurements [14]. It is perhaps not surprising, though undeniably unfortunate, that their prescient proposal was ignored by the wider community and has remained dormant until today.

Diffraction-limited image recovery from bispectral data obtained using such partially redundant masks (PRM) is now routine, and has been demonstrated at both high and low light levels ( 0.5 photons/coherence area/interferogram) to achieve dynamic ranges $(\geq 50: 1)$ characteristic of non-redundant masking [13]. The success of these preliminary experiments suggests that this sort of hybrid approach, intermediate between speckle and NRM, may indeed represent an ideal imaging strategy for a wide range of astrophysical applications - my hope is that future simulations and experiments will verify this possibility.

\section{Acknowledgements}

It is a pleasure to thank the numerous colleagues and collaborators who have contributed to my understanding of interferometric imaging over the years, in particular Dave Buscher. I am grateful to the Royal Society for financial support, without which my attendance at this meeting would have been impossible.

\section{References}

[1] See. e.g., 1988, Proc NOAO-ESO Conference on 'High-resolution imaging by interferometry' Merkle, F. (ed) (ESO:Garching bei München). 
[2] Kulkarni, S.R.: 1988, 'The non-redundant mask technique: theory and practice', Proc NOAO-ESO Conference on 'High-resolution imaging by interferometry' Merkle, F. (ed) (ESO:Garching bei München) 595.

[3] Pearson, T.J and Readhead, A.C.S.: 1984, 'Image formation by self-calibration in radio astronomy', Ann. Rev. Astron. Astrophys. 22, 97.

[4] Wieringa, M.H.: 1992, 'An investigation of the telescope based calibration methods redundancy and self-cal', Experimental A stronomy 2, 203.

[5] Bartelt, H., Lohmann, A.W., and Wirnitzer, B.: 1984, 'Phase and amplitude recovery from the bispectrum', Applied Optics 23, 3121.

[6] Lannes, A.: 1989, 'Backprojection mechanisms in phase-closure imaging: bispectral analysis of the phase-restoration process', Experimental Astronomy 1, 47.

[7] See. e.g., Roddier, F.: 1988, 'Interferometric imaging in optical astronomy', Physics Reports $170,97$.

[8] Roddier, F. and Mariotti, J.-M.; 1993, 'Aperture-plane Interferometry', SPIE-ERIM Infrared and Electro-optics Handbook 8 , in press.

[9] Buscher, D.F.: 1988, 'Aperture masking and speckle masking: a comparison of their signalto-noise ratios', Proc NOAO-ESO Conference on 'High-resolution imaging by interferometry' Merkle, F. (ed) (ESO:Garching bei München) 613.

[10] Kulkarni, S.R., Prasad, S., and Nakajima, T.: 1991, 'Noise in optical synthesis images. II. Sensitivity of an ${ }^{n} C_{2}$ interferometer with bispectrum imaging', J. Opt. Soc. Am. A 8, 499.

[11] See. e.g., Buscher, D.F.: 1993, 'Direct Maximum-Entropy image reconstruction from the bispectrum', these proceedings .

[12] Haniff, C.A. and Buscher, D.F.: 1992, 'Diffraction-limited with partially redundant masks 1. Infrared imaging of bright objects', J. Opt. Soc. Am. A 9, 203.

[13] Buscher, D.F. and Haniff, C.A.: 1993, 'Diffraction-limited with partially redundant masks 2. Optical imaging of faint sources', J. Opt. Soc. Am. A in press.

[14] Aime, C. and Roddier, F.: 1977, 'One-dimensional stellar and solar speckle interferometry', Opt. Commun. 21, 435.

\section{Discussion:}

Ekers:

Full uv coverage is not the same as full aperture. You could have an almost nonredundant mask with full uv coverage.

Haniff:

Absolutely! One could consider a thin annular aperture - this would certainly give full uv coverage, but is not a filled pupil. However its low redundancy would not be advantageous for low light level speckle imaging.

\section{Bedding:}

Why hasn't speckle achieved the $\mathrm{S} / \mathrm{N}$ expected?

Haniff:

Assuming you mean sensitivity at very low light levels, I think that the equations are not lying but that at these signal levels, the signal-to-noise ratios of power and bispectral measurements are just too low to be used effectively in image reconstruction. It is interesting to note that in FFA imaging simulations photon rates of $>1$ photon/coherence area/interferogram are usually required to recover images of 'complex' sources, so in a sense the 'poor' performance of speckle in real life is not unexpected. 